\title{
Delayed Parosmia Following SARS-CoV-2 Infection: a Rare Late Complication of COVID-19
}

\author{
Murat Duyan $^{1} \cdot$ Ibrahim Ulas Ozturan ${ }^{2}$ (D) Murat Altas $^{3}$ \\ Accepted: 23 March 2021 / Published online: 27 March 2021 \\ (C) The Author(s), under exclusive licence to Springer Nature Switzerland AG 2021
}

\begin{abstract}
In this report, we present two cases of delayed parosmia, a rare complication that occurs in the late period after COVID-19. A 28year-old male and a 32-year-old female, found to be positive in rRT-PCR tests for SARS-CoV-2, had a loss of taste and smell, respectively. Following the regaining of their ability to smell after 87 and 72 days, respectively, the male patient reported that the smell he perceived felt the smell of burnt rubber, while the female patient stated that it was similar to the smell of onion. As a result of evaluations, a diagnosis of delayed parosmia was made occurring in the late period secondary to SARS-CoV-2 infection. Although anosmia is a common symptom in the early phase of COVID-19, olfactory damage due to Sars-CoV-2 can be persistent, and distorted sense of smell can be prolonged.
\end{abstract}

Keywords Parosmia $\cdot$ SARS-CoV-2 $\cdot$ COVID-19 $\cdot$ Olfactory dysfunction

\section{Introduction}

Although COVID-19 is predominantly associated with fever, fatigue, generalized body ache, and pulmonary symptoms, smell and taste disorders are also common in the initial presentation of the patients [1]. Moreover, olfactory dysfunction may be the only symptom in mild disease [2]. Although it usually lasts around 1 to 3 weeks in COVID-19 [3], prolonged parosmia exceeding 10 weeks was not previously reported.

While parosmia, one of the olfactory dysfunctions, characterized by a misperception of existing odors, is more frequently detected in patients with postinfectious olfactory dysfunction, it also may occur secondary to head trauma, sinonasal abnormalities, neurodegenerative diseases, and idiopathic causes. The patients previously with a perfectly normal sense of smell reported that they typically perceived all the smell

This article is part of the Topical Collection on Covid-19

Ibrahim Ulas Ozturan

ozturan.iu@gmail.com

1 Department of Emergency Medicine, Antalya Training and Research Hospital, Antalya, Turkey

2 Department of Emergency Medicine, Mersin Toros State Hospital, Mersin, Turkey

3 Department of Neurosurgery, Akdeniz University Faculty of Medicine, Antalya, Turkey sensations as a dirty, rotten, sewage, or burning smell [4]. Viruses can lead to olfactory dysfunction by causing damage to the upper respiratory tract and olfactory neurons [5]. The mechanism underlying the olfactory dysfunction in COVID19 is believed that olfactory epithelium cells express two receptors, ACE2 and TMPRSS2 proteases, which facilitate SARS-CoV-2 binding, replication, and accumulation [6].

In this report, we present two cases of delayed parosmia which is a rare complication after Sars-CoV-2 infection.

\section{Case Presentation}

\section{Case 1}

A 28-year-old male patient was admitted to the emergency department (ED) on July 6, 2020, with complaints of chills, fever, mild shortness of breath, myalgia, and cough. He had no other additional complaints, allergies, or relevant past medical history. His vital signs were a temperature of $37.9^{\circ} \mathrm{C}$, $\mathrm{SpO} 2$ of $98 \%$, a pulse of 94 beats/min, respiratory rate of $22 / \mathrm{min}$, and arterial blood pressure of $140 / 80 \mathrm{mmHg}$. Electrocardiography (ECG) showed a normal sinus rhythm. There was no pneumonia in chest computerized tomography (CT). The rRT-PCR from the nasopharyngeal swab was positive for SARS-CoV-2. Hydroxychloroquine $400 \mathrm{mg}$ per oral (po) (200 mg twice a day) daily for 5 days was administrated. 
Two days after the diagnosis, the taste and smell sensations were completely lost. After 14 days of isolation, the patient had negative rRT-PCR test and started to regain his normal sense of taste about 53 days after COVID-19, but the loss of smell continued. The patient regained the sense of smell on 87 th day of the infection. However, the patient reported the all the smells he perceived had distorted, similar to the smell of burnt rubber.

\section{Case 2}

A 32-year-old female patient was admitted to the ED on July 7, 2020, with no additional complaints other than myalgia and fatigue. She had no allergies or relevant past medical history. Her vital signs were normal. Initial nasopharyngeal RT-PCR test was negative. Due to the history of high-risk contact, hydroxychloroquine $400 \mathrm{mg}$ po (200 mg twice a day) daily for 5 days was empirically administrated. On July 13, 2020, she was admitted to the ED again due to anosmia, loss of taste, and mild shortness of breath. In the chest CT, parenchymal infiltration typical for early findings of COVID-19 was detected. ECG showed a normal sinus rhythm. The rRT-PCR test from the nasopharyngeal swab was positive. Favipravir was administered with a loading dose of $1600 \mathrm{mg}$ twice a day on day 1 and then $600 \mathrm{mg}$ twice a day for another 4 days. After the treatment, other symptoms, apart from taste and smell dysfunction, improved. She started to taste and smell after about 72 days. However, she noticed that everything that she smells felt her of the smell of onion.

Both cases had smell and taste dysfunction without rhinorrhea or nasal obstruction. Following the exclusion of the secondary causes for parosmia with multiparametric olfactory functional magnetic resonance imaging (MRI), CT of brain and paranasal sinus, and psychiatric examinations, delayed parosmia due to Sars-Cov2 was confirmed. Both patients were referred to smell training for olfactory function loss.

\section{Discussion}

As the SARS-CoV-2 outbreak continues to affect the globe, it should be noted that rare complications might emerge in the late period and we need a better recognition of the associated symptoms. In both of our cases, there was an anosmia and a lack of taste in the early period. Interestingly, parosmia was not seen in the early period. It appeared after 87 days in the male patient and 72 days in the female patient. In an article from 2005, it was stated that the duration of parosmia ranged from 3 months to 22 years on average. In addition, in the same article, the onset of parosmia started within 3 months after the smell disorder in 19 patients and after 3 months in 5 patients
[7]. In a case report, parosmia lasting 6 months after upper respiratory tract infection (URTI) was also reported [8].

The reason why parosmia appears in the late period may be due to persistent degeneration of olfactory receptors after infection with SARS-CoV-2 or the low number of partially healing neurons. Disorganized renovation of immature neurons had been reported to be associated with parosmia, however is expected to occur as an early symptom in COVID-19 patients [9]. In a report, delayed neuropsychiatric and neurodegenerative complication of SARS-CoV-2 infection was evaluated. 18F-FDG PET hypometabolism in the olfactory/ rectus gyrus was detected in 2 patients, especially in one with 4 weeks of prolonged anosmia. It was hypothesized that SARS-CoV-2 neurotropism through the olfactory bulb could be the potential cause of delayed neuropsychiatric and neurodegenerative complications in COVID-19 patients [10].

There is not enough data in the literature regarding how long the parosmia that occurs in the late period in COVID19. When parosmia developed in both of our cases, there was no rhinorrhea or nasal obstruction. In our cases, secondary causes were excluded by MRI, brain and paranasal sinus $\mathrm{CT}$, and psychiatric examinations. We continue the followups in both patients because the symptom of parosmia still persists.

\section{Conclusion}

With the SARS-CoV-2 pandemic still continuing, rare complications continue to be identified. Anosmia usually persists for several weeks after the patient has recovered from COVID-19. However, there is not enough data about the period of parosmia that occurs in the late period and how long it lasts. In light of our report, we recommend that COVID-19 patients with odor dysfunction should be followed up as they might have symptoms that may arise in the late period. If parosmia is detected, they should be referred to otolaryngologists for scent training.

Code Availability Not applicable.

Authors' Contributions MD and MA were the treating physicians. MD and MA collected the data. MD, IUO, and MA conceptualized the report. IUO drafted manuscript. MD, IUO, and MA critically reviewed the manuscript. All authors approved the final version.

Data availability Not applicable.

\section{Declarations}

Ethics Approval IRB exempt.

Consent to Participate Not applicable. 
Consent for Publication Informed consent was obtained from the patients for this case report.

Conflict of Interest The authors declare no competing interests.

\section{References}

1. Zhu N, Zhang D, Wang W, et al. A novel coronavirus from patients with pneumonia in China, 2019. N Engl J Med. 2020;382(8).

2. Lechien JR, Chiesa-Estomba CM, De Siati DR, et al. Olfactory and gustatory dysfunctions as a clinical presentation of mild-tomoderate forms of the coronavirus disease (COVID-19): a multicenter European study. Eur Arch Oto-Rhino-Laryngology. 2020;277(8):2251-61.

3. Lee Y, Min P, Lee S, Kim SW. Prevalence and duration of acute loss of smell or taste in COVID-19 patients. J Korean Med Sci. 2020;35(18):e174.

4. Ferrier MB, Spuesens EB, Le Cessie S, Baatenburg de Jong RJ. Comorbidity as a major risk factor for mortality and complications in head and neck surgery. Arch Otolaryngol Neck Surg. 2005;131(1).
5. Liu D, Sabha M, Damm M, et al. Parosmia is associated with relevant olfactory recovery after olfactory training. The Laryngoscope. 2020;131:618-23.

6. Bilinska K, Jakubowska P, Von Bartheld CS, Butowt R. Expression of the SARS-CoV-2 entry proteins, ACE2 and TMPRSS2, in cells of the olfactory epithelium: identification of cell types and trends with age. ACS Chem Neurosci. 2020;11(11).

7. Bonfils P, Avan P, Faulcon P, Malinvaud D. Distorted odorant perception. Arch Otolaryngol Neck Surg. 2005;131(2).

8. Landis BN, Frasnelli J, Hummel T. Euosmia: a rare form of parosmia. Acta Otolaryngol. 2006;126(1).

9. Saussez S, Lechien JR, Hopkins C. Anosmia: an evolution of our understanding of its importance in COVID-19 and what questions remain to be answered. Eur Arch Otorhinolaryngol. 2020. https:// doi.org/10.1007/s00405-020-06285-0.

10. Guedj E, Million M, Dudouet P, Tissot-Dupont H, Bregeon F, Cammilleri S, et al. 18F-FDG brain PET hypometabolism in postSARS-CoV-2 infection: substrate for persistent/delayed disorders? Eur J Nucl Med Mol Imaging. 2021;48:592-5.

Publisher's Note Springer Nature remains neutral with regard to jurisdictional claims in published maps and institutional affiliations. 